\title{
Geometric Mesh Three-Point Discretization for Fourth-Order Nonlinear Singular Differential Equations in Polar System
}

\author{
Navnit Jha, ${ }^{1}$ R. K. Mohanty, ${ }^{1,2}$ and Vinod Chauhan ${ }^{2}$ \\ ${ }^{1}$ Department of Mathematics, South Asian University, Akbar Bhawan, Chanakya Puri, New Delhi 110021, India \\ ${ }^{2}$ Department of Mathematics, Faculty of Mathematical Sciences, University of Delhi, Delhi 110007, India
}

Correspondence should be addressed to Navnit Jha; navnitjha@sau.ac.in

Received 9 June 2013; Revised 8 August 2013; Accepted 23 August 2013

Academic Editor: Rüdiger Weiner

Copyright (c) 2013 Navnit Jha et al. This is an open access article distributed under the Creative Commons Attribution License, which permits unrestricted use, distribution, and reproduction in any medium, provided the original work is properly cited.

Numerical method based on three geometric stencils has been proposed for the numerical solution of nonlinear singular fourthorder ordinary differential equations. The method can be easily extended to the sixth-order differential equations. Convergence analysis proves the third-order convergence of the proposed scheme. The resulting difference equations lead to block tridiagonal matrices and can be easily solved using block Gauss-Seidel algorithm. The computational results are provided to justify the usefulness and reliability of the proposed method.

\section{Introduction}

Consider the fourth-order boundary value problem:

$$
\begin{array}{r}
u^{(4)}(r)=f\left(r, u(r), u^{(1)}(r), u^{(2)}(r), u^{(3)}(r)\right), \\
\alpha<r<\beta
\end{array}
$$

subject to the necessary boundary conditions:

$$
\begin{array}{ll}
u(\alpha)=\alpha_{0}, & u^{(2)}(\alpha)=\alpha_{1}, \\
u(\beta)=\beta_{0}, & u^{(2)}(\beta)=\beta_{0},
\end{array}
$$

where $f \in C^{4}[\alpha, \beta]$ and $\alpha_{0}, \alpha_{1}, \beta_{0}$, and $\beta_{1}$ are real constants and $-\infty<\alpha<r<\beta<\infty$.

Or equivalently

$$
\begin{gathered}
u^{(2)}(r)=v(r), \\
v^{(2)}(r)=f\left(r, u(r), u^{(1)}(r), v(r), v^{(1)}(r)\right), \\
\alpha<r<\beta
\end{gathered}
$$

subject to the natural boundary conditions:

$$
\begin{array}{ll}
u(\alpha)=\alpha_{0}, & v(\alpha)=\alpha_{1}, \\
u(\beta)=\beta_{0}, & v(\beta)=\beta_{1} .
\end{array}
$$

Fourth-order differential equations occur in various areas of mathematics such as viscoelastic and inelastic flows, beam theory, Lifshitz point in phase transition physics (e.g., nematic liquid crystal, crystals, and ferroelectric crystals) [1], the rolls in a Rayleigh-Benard convection cell (two parallel plates of different temperature with a liquid in between) [2], spontaneous pattern formation in second-order materials (e.g., polymeric fibres) [3], the waves on a suspension bridge $[4,5]$, geological folding of rock layers [6], buckling of a strut on a nonlinear elastic foundation [7], traveling water waves in a shallow channel [8], pulse propagation in optical fibers [9], system of two reaction diffusion equation [10], and so forth.

The existence and uniqueness of the solution for the fourth and higher-order boundary value problems have been discussed in [11-14]. In the recent past, the numerical solution of fourth-order differential equations has been developed using multiderivative, finite element method, Ritz method, spline collocation, and finite difference method [15-18]. The determination of eigen values of self adjoint fourthorder differential equations was developed in [19] using finite difference scheme. The motivation of variable mesh technique for differential equations arises from the theory of electrochemical reaction-convection-diffusion problems in one-dimensional space geometry [20]. The geometric mesh method for self-adjoint singular perturbation problems using 
finite difference approximations was discussed in [21]. The use of geometric mesh in the context of boundary value problems was studied extensively in [22-24]. In this paper, we derive a geometric mesh finite difference method for the solution of fourth- and sixth-order differential boundary value problems with order of accuracy being three. The simplicity of the proposed method lies in its three-point discretization without any use of fictitious nodes. The scheme is compact and applicable to both singular and nonsingular problems. The resulting difference equations are solved using block Gauss-Seidel algorithm for linear case, and corresponding Newton's method has been applied to nonlinear problems.

The paper is outlined in the following manner: in Section 2, the derivation of the method is discussed in detail. In Section 3, we define the procedure for numerical solution to singular problems in such a way that the method retains the order and accuracy even in the vicinity of singularity. In Section 4, algorithmic details are provided for the numerical solution of sixth-order differential equations. The convergence property has been discussed briefly in Section 4 . The numerical illustrations based on geometric mesh as well as uniform mesh were provided in Section 5. The paper is concluded in the last section with future development and remarks.

\section{Derivation of the Numerical Scheme}

We discretize the solution region $[\alpha, \beta]$ such that $\alpha=r_{0}<$ $r_{1}<\cdots<r_{n}<r_{n+1}=\beta$. Let $h_{k}=r_{k}-r_{k-1}$ and $k=1(1) n+1$ be the nonuniform step size, and let $\sigma_{k}=h_{k+1} / h_{k}>0$ be the geometric mesh ratio. Let $U_{k}=u\left(r_{k}\right)$ and $V_{k}=v\left(r_{k}\right)$ denote the exact solution values of $u$ and $v$ at the mesh $r_{k}$ and $u_{k}, v_{k}$ be their approximate solutions, respectively.

Consider the following three-point geometric mesh discretizations for $u^{(2)}(r)=v(r)$ and $v^{(2)}(r)=f(r)$ :

$$
\begin{aligned}
& U_{k+1}-\left(1+\sigma_{k}\right) U_{k}+\sigma_{k} U_{k-1}+\frac{h^{2}}{12}\left(p_{k} V_{k+1}+q_{k} V_{k}+r_{k} V_{k-1}\right) \\
& =O\left(h_{k}^{5}\right), \quad k=1(1) n, \\
& V_{k+1}-\left(1+\sigma_{k}\right) V_{k}+\sigma_{k} V_{k-1}+\frac{h^{2}}{12}\left(p_{k} f_{k+1}+q_{k} f_{k}+r_{k} f_{k-1}\right) \\
& =E_{k}, \quad k=1(1) n,
\end{aligned}
$$

where

$$
\begin{aligned}
E_{k}= & \frac{h_{k}^{5}}{360} \sigma_{k}\left(2 \sigma_{k}+1\right)\left(\sigma_{k}+2\right)\left(1-\sigma_{k}^{2}\right) f_{k}^{(5)}(\xi) \\
& +O\left(h_{k}^{6}\right), \quad r_{k-1}<\xi<r_{k+1}, \\
p_{k}= & 1-\sigma_{k}-\sigma_{k}^{2}, \\
q_{k}= & -\left(\sigma_{k}+1\right)\left(\sigma_{k}^{2}+3 \sigma_{k}+1\right), \\
r_{k}= & \sigma_{k}\left(\sigma_{k}^{2}-\sigma_{k}-1\right) .
\end{aligned}
$$

Define

$$
\begin{gathered}
\widetilde{U}_{k}^{\prime}=\frac{U_{k+1}-\left(1-\sigma_{k}^{2}\right) U_{k}-\sigma_{k}^{2} U_{k-1}}{h_{k} \sigma_{k}\left(1+\sigma_{k}\right)}, \\
\widetilde{V}_{k}^{\prime}=\frac{V_{k+1}-\left(1-\sigma_{k}^{2}\right) V_{k}-\sigma_{k}^{2} V_{k-1}}{h_{k} \sigma_{k}\left(1+\sigma_{k}\right)}, \\
\widetilde{U}_{k+1}^{\prime}=\frac{\left(1+2 \sigma_{k}\right) U_{k+1}-\left(1+\sigma_{k}\right)^{2} U_{k}+\sigma_{k}^{2} U_{k-1}}{h_{k} \sigma_{k}\left(1+\sigma_{k}\right)}, \\
\widetilde{V}_{k+1}^{\prime}=\frac{\left(1+2 \sigma_{k}\right) V_{k+1}-\left(1+\sigma_{k}\right)^{2} V_{k}+\sigma_{k}^{2} V_{k-1}}{h_{k} \sigma_{k}\left(1+\sigma_{k}\right)} \\
\widetilde{U}_{k-1}^{\prime}=\frac{-U_{k+1}+\left(1+\sigma_{k}\right)^{2} U_{k}-\sigma_{k}\left(2+\sigma_{k}\right) U_{k-1}}{h_{k} \sigma_{k}\left(1+\sigma_{k}\right)}, \\
\widetilde{V}_{k-1}^{\prime}=\frac{-V_{k+1}+\left(1+\sigma_{k}\right)^{2} V_{k}-\sigma_{k}\left(2+\sigma_{k}\right) V_{k-1}}{h_{k} \sigma_{k}\left(1+\sigma_{k}\right)} \\
\widetilde{f}_{k \pm 1}=f\left(r_{k \pm 1}, U_{k \pm 1}, \widetilde{U}_{k \pm 1}^{\prime}, V_{k \pm 1}, \widetilde{V}_{k \pm 1}^{\prime}\right) .
\end{gathered}
$$

It is easy to verify that

$$
\begin{aligned}
& \tilde{f}_{k+1}=f_{k+1}-\frac{h_{k}^{2}}{6} \sigma_{k}\left(\sigma_{k}+1\right)\left(G_{k} U_{k}^{(3)}+H_{k} V_{k}^{(3)}\right)+O\left(h_{k}^{3}\right), \\
& \tilde{f}_{k-1}=f_{k-1}-\frac{h_{k}^{2}}{6}\left(\sigma_{k}+1\right)\left(G_{k} U_{k}^{(3)}+H_{k} V_{k}^{(3)}\right)+O\left(h_{k}^{3}\right),
\end{aligned}
$$

where $G_{k}=\left(\partial f / \partial U^{(1)}\right)_{r_{k}}, H_{k}=\left(\partial f / \partial V^{(1)}\right)_{r_{k}}$, and so forth.

Now, let

$$
\begin{aligned}
\widehat{U}_{k}^{\prime} & =\widetilde{U}_{k}^{\prime}+\gamma h_{k}\left(V_{k+1}-V_{k-1}\right), \\
\widehat{V}_{k}^{\prime} & =\widetilde{V}_{k}^{\prime}+\delta h_{k}\left(\widetilde{f}_{k+1}-\widetilde{f}_{k-1}\right),
\end{aligned}
$$

where $\gamma$ and $\delta$ are free parameters to be determined.

With the help of (8) and (11), it follows that

$$
\begin{aligned}
& \widehat{U}_{k}^{\prime}=U_{k}^{(1)}+\frac{h_{k}^{2}}{6}\left(\sigma_{k}+6 \gamma\left(1+\sigma_{k}\right)\right) U_{k}^{(3)}+O\left(h_{k}^{3}\right), \\
& \widehat{V}_{k}^{\prime}=V_{k}^{(1)}+\frac{h_{k}^{2}}{6}\left(\sigma_{k}+6 \delta\left(1+\sigma_{k}\right)\right) V_{k}^{(3)}+O\left(h_{k}^{3}\right) .
\end{aligned}
$$

Further, we define

$$
\widehat{f}_{k}=f\left(r_{k}, U_{k}, \widehat{U}_{k}^{\prime}, V_{k}, \widehat{V}_{k}^{\prime}\right) .
$$

With the help of (12)-(13), it follows that

$$
\begin{aligned}
\widehat{f}_{k}= & f_{k}+\frac{h_{k}^{2}}{6}\left(\sigma_{k}+6 \gamma\left(1+\sigma_{k}\right)\right) G_{k} U_{k}^{(3)} \\
& +\frac{h_{k}^{2}}{6}\left(\sigma_{k}+6 \delta\left(1+\sigma_{k}\right)\right) H_{k} V_{k}^{(3)}+O\left(h_{k}^{3}\right) .
\end{aligned}
$$


With the help of approximations (10), (14), the difference equation (6) at each internal mesh $r_{k}$, is approximated as follows:

$$
\begin{aligned}
V_{k+1} & -\left(1+\sigma_{k}\right) V_{k}+\sigma_{k} V_{k-1}+\frac{h_{k}^{2}}{12}\left(p_{k} \widetilde{f}_{k}+q_{k} \widehat{f}_{k}+r_{k} \widetilde{f}_{k-1}\right) \\
& =\widetilde{E}_{k}, \quad k=1(1) n,
\end{aligned}
$$

where

$$
\begin{aligned}
\widetilde{E}_{k}= & -\frac{h_{k}^{4}}{72} U_{k}^{(3)} G_{k}\left(1+\sigma_{k}\right)\left(6 \gamma+(1+24 \gamma) \sigma_{k}\left(1+\sigma_{k}\right)\right. \\
& \left.+(1+6 \gamma) \sigma_{k}^{3}\right)-\frac{h_{k}^{4}}{72} V_{k}^{(3)} H_{k} \\
& \times\left(1+\sigma_{k}\right)\left(6 \delta+(1+24 \delta) \sigma_{k}+(1+6 \delta) \sigma_{k}^{3}\right) \\
& +O\left(h_{k}^{5}\right), \quad \sigma_{k} \neq 1 .
\end{aligned}
$$

The difference scheme (15) to be of $O\left(h_{k}^{3}\right)$, the coefficients of $h_{k}^{4}$ in (16) must be zero, and hence we obtain

$$
\begin{aligned}
& 6 \gamma+(1+24 \gamma) \sigma_{k}\left(1+\sigma_{k}\right)+(1+6 \gamma) \sigma_{k}^{3}=0 \\
& 6 \delta+(1+24 \delta) \sigma_{k}\left(1+\sigma_{k}\right)+(1+6 \delta) \sigma_{k}^{3}=0
\end{aligned}
$$

Thus the values associated with (17) are given by $\gamma=\delta=$ $-\sigma_{k}\left(\sigma_{k}^{2}+\sigma_{k}+1\right) /\left(6\left(1+\sigma_{k}\right)\left(\sigma_{k}^{2}+3 \sigma_{k}+1\right)\right)$, and the local truncation errors given by (16) becomes $\widetilde{E}_{k}=O\left(h_{k}^{5}\right), \sigma_{k} \neq 1$. However, for $\sigma_{k} \neq 1$, the error reduces to $\widetilde{E}_{k}=O\left(h_{k}^{6}\right)$.

\section{Application to Singular Problem}

Consider the singular fourth-order linear differential equation in cylindrical polar coordinates:

$$
\Delta^{4} u(r) \equiv\left(\frac{d^{2}}{d r^{2}}+\frac{\lambda}{r} \frac{d}{d r}\right)^{2} u(r)=g(r), \quad 0<r<1
$$

or equivalently

$$
\begin{gathered}
u^{(2)}(r)=v(r), \\
v^{(2)}(r)=a(r) v^{(1)}(r)+b(r) v(r)+c(r) u^{(1)}(r)+g(r),
\end{gathered}
$$

where $a(r)=-2 \lambda / r, b(r)=-\lambda(\lambda-2) / r^{2}$, and $c(r)=$ $\lambda(\lambda-2) / r^{3}$ are the singular coefficients. The associated boundary conditions are given by (4). For $\lambda=0$, 1 , or 2 , the differential equation (18) shows planar, cylindrical, or spherical geometries (see, $[25,26]$ ).
Applying the difference schemes (5) and (15) to (19) and (20), respectively, we obtain a system of coupled difference equations for $k=1(1) n$ :

$$
\begin{gathered}
{\left[\begin{array}{cc}
s b_{k}^{11} & s b_{k}^{12} \\
s b_{k}^{21} & s b_{k}^{22}
\end{array}\right]\left[\begin{array}{l}
U_{k-1} \\
V_{k-1}
\end{array}\right]+\left[\begin{array}{ll}
d g_{k}^{11} & d g_{k}^{12} \\
d g_{k}^{21} & d g_{k}^{22}
\end{array}\right]\left[\begin{array}{l}
U_{k} \\
V_{k}
\end{array}\right]} \\
+\left[\begin{array}{cc}
s p_{k}^{11} & s p_{k}^{12} \\
s p_{k}^{21} & s p_{k}^{22}
\end{array}\right]\left[\begin{array}{l}
U_{k+1} \\
V_{k+1}
\end{array}\right]=\left[\begin{array}{c}
R_{k}^{11} \\
R_{k}^{21}
\end{array}\right],
\end{gathered}
$$

where

$$
\begin{aligned}
& s b_{k}^{11}=\sigma_{k}, \quad s p_{k}^{11}=1, \\
& d g_{k}^{11}=-1-\sigma_{k}, \quad s b_{k}^{12}=\frac{h_{k}^{2}}{12} r_{k}, \\
& s p_{k}^{12}=\frac{h_{k}^{2}}{12} p_{k}, \quad d g_{k}^{12}=\frac{h_{k}^{2}}{12} q_{k}, \quad R_{k}^{11}=0 \text {, } \\
& s b_{k}^{21}=\frac{h_{k}}{12\left(1+\sigma_{k}\right)} \\
& \times\left(\gamma h_{k} q_{k} a_{k}\left(\sigma_{k} c_{k+1}+\left(2+\sigma_{k}\right)\left(a_{k-1}+c_{k-1}\right)\right)\right. \\
& \left.-\sigma_{k}\left(q_{k} c_{k}-p_{k} c_{k+1}\right)+\left(2+\sigma_{k}\right) r_{k}\left(a_{k-1}+c_{k-1}\right)\right) \text {, } \\
& s p_{k}^{21}=\frac{h_{k}}{12 \sigma_{k}\left(1+\sigma_{k}\right)} \\
& \times\left(\gamma h_{k} q_{k} a_{k}\left(\left(2 \sigma_{k}+1\right) c_{k+1}+c_{k-1}\right)\right. \\
& \left.+\left(2 \sigma_{k}+1\right) c_{k+1} p_{k}+c_{k} q_{k}-r_{k} c_{k-1}\right), \\
& d g_{k}^{21}=\frac{h_{k}}{12 \sigma_{k}}\left(\sigma_{k}\left(q_{k} c_{k}-p_{k} c_{k+1}+r_{k} c_{k-1}\right)\right. \\
& -c_{k+1} p_{k}+r_{k} c_{k-1}-c_{k} q_{k} \\
& \left.-\gamma h_{k} q_{k} a_{k}\left(1+\sigma_{k}\right)\left(c_{k+1}+c_{k-1}\right)\right), \\
& s b_{k}^{22}=\sigma_{k}-\frac{\gamma h_{k}^{3}}{12} q_{k}\left(a_{k} b_{k-1}+c_{k}\right) \\
& +\frac{h_{k}}{12\left(1+\sigma_{k}\right)}\left(h_{k}\left(\gamma q_{k} a_{k} a_{k+1} \sigma_{k}+\left(1+\sigma_{k}\right) r_{k} b_{k-1}\right)\right. \\
& \left.+\sigma_{k}\left(p_{k} a_{k+1}-a_{k} q_{k}\right)\right), \\
& s p_{k}^{22}=1+\frac{\gamma h_{k}^{3}}{12} q_{k}\left(a_{k} b_{k+1}+c_{k}\right)+\frac{h_{k}}{12 \sigma_{k}\left(1+\sigma_{k}\right)} \\
& \times\left(h _ { k } \left(\gamma q_{k} a_{k}\left(\left(2 \sigma_{k}+1\right) a_{k+1}+a_{k-1}\right)\right.\right. \\
& \left.+\sigma_{k}\left(\sigma_{k}+1\right) p_{k} b_{k+1}\right) \\
& \left.+\left(1+2 \sigma_{k}\right) p_{k} a_{k+1}-r_{k} a_{k-1}+a_{k} q_{k}\right),
\end{aligned}
$$




$$
\begin{aligned}
d g_{k}^{22}=-1-\sigma_{k} & \\
+\frac{h_{k}}{12 \sigma_{k}}( & \left(\sigma_{k}\left(q_{k} a_{k}-p_{k} a_{k+1}+r_{k} a_{k-1}\right)\right. \\
& \left.\quad-p_{k} a_{k+1}+r_{k} a_{k-1}-a_{k} q_{k}\right) \\
& \left.-h_{k} q_{k}\left(\gamma a_{k}\left(\sigma_{k}+1\right)\left(a_{k+1}+a_{k-1}\right)-b_{k} \sigma_{k}\right)\right), \\
R_{k}^{21}= & -\frac{\gamma h_{k}^{3}}{12} a_{k} q_{k}\left(g_{k+1}-g_{k-1}\right) \\
& -\frac{h_{k}^{2}}{12}\left(q_{k} g_{k}+p_{k} g_{k+1}+r_{k} g_{k-1}\right) .
\end{aligned}
$$

Note that the scheme (21) fails when the solution is to be determined at $k=1$. We overcome this difficulty by modifying the scheme in such a way that the solutions retain order and accuracy even in the vicinity of singularity $r=0$. We consider the following approximations:

$$
\begin{aligned}
a_{k+1}= & a_{k}+h_{k} \sigma_{k} a_{k}^{(1)}+\frac{h_{k}^{2} \sigma_{k}^{2}}{2 !} a_{k}^{(2)}+\frac{h_{k}^{3} \sigma_{k}^{3}}{3 !} a_{k}^{(3)} \\
& +\frac{h_{k}^{4} \sigma_{k}^{4}}{4 !} a_{k}^{(4)}+O\left(h_{k}^{5}\right), \\
a_{k-1}= & a_{k}-h_{k} a_{k}^{(1)}+\frac{h_{k}^{2}}{2 !} a_{k}^{(2)}-\frac{h_{k}^{3}}{3 !} a_{k}^{(3)} \\
& +\frac{h_{k}^{4}}{4 !} a_{k}^{(4)}+O\left(h_{k}^{5}\right) .
\end{aligned}
$$

Using the similar approximations of $b_{k \pm 1}, c_{k \pm 1}$ and $g_{k \pm 1}$ and neglecting $O\left(h_{k}^{5}\right)$ terms, we can rewrite (21) with the help of compact operators as

$$
\begin{gathered}
{\left[\begin{array}{cc}
s b_{k}^{11} & s b_{k}^{12} \\
\widetilde{s b}_{k}^{21} & \widetilde{s b}_{k}^{22}
\end{array}\right]\left[\begin{array}{l}
U_{k-1} \\
V_{k-1}
\end{array}\right]+\left[\begin{array}{cc}
d g_{k}^{11} & d g_{k}^{12} \\
\widetilde{d g}_{k}^{21} & \widetilde{d g}_{k}^{22}
\end{array}\right]\left[\begin{array}{l}
U_{k} \\
V_{k}
\end{array}\right]} \\
+\left[\begin{array}{cc}
s p_{k}^{11} & s p_{k}^{12} \\
\widetilde{s p}_{k}^{21} & \widetilde{s p}_{k}^{22}
\end{array}\right]\left[\begin{array}{l}
U_{k+1} \\
V_{k+1}
\end{array}\right]=\left[\begin{array}{c}
R_{k}^{11} \\
\widetilde{R}_{k}^{21}
\end{array}\right],
\end{gathered}
$$

where

$$
\begin{aligned}
& \widetilde{s b}_{k}^{21}=\left(\left(12 \gamma q_{k} a_{k} \sigma_{k}\left(\sigma_{k}+1\right)^{2} h_{k}^{2}+6\left(\sigma_{k}+1\right) \sigma_{k}\right.\right. \\
& \left.\times\left(-r_{k} \sigma_{k}+\sigma_{k} p_{k}-q_{k} \sigma_{k}-2 r_{k}\right) h_{k}\right) c_{k} \\
& +\left(-6 \gamma q_{k} a_{k} \sigma_{k}\left(\sigma_{k}+1\right)^{2} h_{k}^{3}+6\left(\sigma_{k}+1\right)\right. \\
& \left.\times \sigma_{k}\left(p_{k} \sigma_{k}^{2}+r_{k} \sigma_{k}+2 r_{k}\right) h_{k}^{2}\right) c_{k}^{(1)}-3\left(\sigma_{k}+1\right) \\
& \times \sigma_{k}\left(p_{k} \sigma_{k}^{2}+r_{k}\right) h_{k}^{3} c_{k}^{(2)}-3 \sigma_{k}\left(\sigma_{k}+2\right) \\
& \times\left(2 \gamma q_{k} a_{k} a_{k}^{(2)}+a_{k}^{(2)} r_{k}\right) h_{k}^{3} \\
& +6 \sigma_{k}\left(\sigma_{k}+2\right)\left(\sigma_{k}+1\right)\left(r_{k} a_{k}^{(1)}+\gamma a_{k}^{2} q_{k}\right) h_{k}^{2} \\
& \left.-6 r_{k} a_{k} \sigma_{k}\left(\sigma_{k}+2\right)\left(\sigma_{k}+1\right) h_{k}\right) \\
& \times\left(72 \sigma_{k}\left(\sigma_{k}+1\right)^{2}\right)^{-1}, \\
& s p_{k}^{21}=\left(6 q_{k} a_{k} \gamma \sigma_{k}\left(\sigma_{k}+2\right) h_{k}^{3} a_{k}^{(1)}\right. \\
& +\left(12 a_{k} \gamma q_{k}\left(\sigma_{k}+1\right)^{2} h_{k}^{2}+6\left(\sigma_{k}+1\right)\right. \\
& \left.\times\left(\left(2 \sigma_{k}+1\right) p_{k}-r_{k}+q_{k}\right) h_{k}\right) c_{k} \\
& +6 h_{k}^{2}\left(\sigma_{k}+1\right)\left(q_{k} a_{k} \gamma \sigma_{k}\left(\sigma_{k}+1\right) h_{k}\right. \\
& \left.+\sigma_{k}\left(2 \sigma_{k}+1\right) p_{k}+r_{k}\right) c_{k}^{(1)} \\
& +3\left(\sigma_{k}+1\right) \sigma_{k}\left(p_{k} \sigma_{k}^{2}+r_{k}\right) h_{k}^{3} c_{k}^{(2)}+3 r_{k} a_{k}^{(2)} \sigma_{k} \\
& \left.\times\left(\sigma_{k}+2\right) h_{k}^{3}\right) \times\left(72 \sigma_{k}\left(1+\sigma_{k}\right)^{2}\right)^{-1}, \\
& \widetilde{d g}_{k}^{21}=\left(\sigma_{k}\left(\sigma_{k}+2\right)\left(\sigma_{k}+1\right)\left(a_{k}^{(3)} r_{k}+3 \gamma q_{k} a_{k} a_{k}^{(2)}\right) h_{k}^{4}\right. \\
& -3 \sigma_{k}\left(\sigma_{k}+2\right)\left(\sigma_{k}+1\right)\left(2 \gamma q_{k} a_{k} a_{k}^{(1)}+a_{k}^{(2)} r_{k}\right) h_{k}^{3} \\
& -6\left(\sigma_{k}+1\right)^{3}\left(p_{k} \sigma_{k} c_{k}^{(1)}+2 \gamma q_{k} a_{k} c_{k}+r_{k} c_{k}^{(1)}\right) h_{k}^{2} \\
& -6 c_{k}\left(\sigma_{k}+1\right)^{2}\left(-r_{k} \sigma_{k}+\sigma_{k} p_{k}-q_{k} \sigma_{k}-r_{k}+q_{k}+p_{k}\right) \\
& \left.\times h_{k}\right) \times\left(72 \sigma_{k}\left(\sigma_{k}+1\right)^{2}\right)^{-1} \text {, } \\
& \widetilde{s b}_{k}^{22}=1+\left(6 \gamma q_{k} \sigma_{k}^{2}\left(\sigma_{k}+1\right) h_{k}^{2} a_{k}^{2}-6 h_{k} \sigma_{k}\right. \\
& \times\left(\gamma h_{k}^{2} q_{k}\left(\left(\sigma_{k}^{2}-1+\sigma_{k}\right) a_{k}^{(1)}+b_{k}\left(\sigma_{k}+1\right)^{2}\right)\right. \\
& \left.-\sigma_{k}\left(\sigma_{k}+1\right)\left(p_{k}-q_{k}\right)\right) a_{k}+6 p_{k} \sigma_{k}^{3} \\
& \times\left(\sigma_{k}+1\right) h_{k}^{2} a_{k}^{(1)}-3 \sigma_{k}\left(p_{k} \sigma_{k}^{3}+p_{k} \sigma_{k}^{2}-r_{k}\right) \\
& \times h_{k}^{3} a_{k}^{(2)}-6 \sigma_{k}\left(\sigma_{k}+1\right)\left(p_{k} \sigma_{k}^{2}+r_{k}\right) h_{k}^{3} b_{k}^{(1)} \\
& \left.+6 h_{k}^{2} \sigma_{k}\left(\sigma_{k}+1\right)^{2}\left(r_{k} b_{k}-\gamma q_{k} c_{k} h_{k}\right)\right) \\
& \times\left(72 \sigma_{k}^{2}\left(\sigma_{k}+1\right)^{2}\right)^{-1} \text {, }
\end{aligned}
$$




$$
\begin{aligned}
& \widetilde{s p}_{k}^{22}=1+\left(12 q_{k} \gamma\left(\sigma_{k}+1\right)^{2} h_{k}^{2} a_{k}^{2}\right. \\
& +\left(6 q_{k} \gamma \sigma_{k}\left(\sigma_{k}^{2}-1+\sigma_{k}\right) h_{k}^{3} a_{k}^{(1)}\right. \\
& +6 q_{k} \gamma b_{k} \sigma_{k}\left(\sigma_{k}+1\right)^{2} h_{k}^{3} \\
& \left.+6\left(\sigma_{k}+1\right)\left(2 \sigma_{k} p_{k}-r_{k}+q_{k}+p_{k}\right) h_{k}\right) a_{k} \\
& +6\left(\sigma_{k}+1\right)\left(2 p_{k} \sigma_{k}^{2}+\sigma_{k} p_{k}+r_{k}\right) \\
& \times h_{k}^{2} a_{k}^{(1)}+3 \sigma_{k}\left(p_{k} \sigma_{k}^{3}+p_{k} \sigma_{k}^{2}-r_{k}\right) h_{k}^{3} a_{k}^{(2)} \\
& +6\left(\sigma_{k}+1\right) \sigma_{k}\left(p_{k} \sigma_{k}^{2}+r_{k}\right) h_{k}^{3} b_{k}^{(1)} \\
& +6 q_{k} c_{k} \gamma \sigma_{k}\left(\sigma_{k}+1\right)^{2} h_{k}^{3}+6 p_{k} \sigma_{k} b_{k} \\
& \left.\times\left(\sigma_{k}+1\right)^{2} h_{k}^{2}\right) \times\left(72 \sigma_{k}\left(\sigma_{k}+1\right)^{2}\right)^{-1}, \\
& \widetilde{d g}_{k}^{22}=-1-\sigma_{k} \\
& +\left(-12 \gamma\left(\sigma_{k}+1\right)^{3} q_{k} h_{k}^{2} a_{k}^{2}\right. \\
& +\left(6 \gamma \sigma_{k}\left(\sigma_{k}+2\right)\left(\sigma_{k}+1\right) q_{k} h_{k}^{3} a_{k}^{(1)}\right. \\
& -3 \gamma \sigma_{k}\left(\sigma_{k}+2\right)\left(\sigma_{k}+1\right) q_{k} h_{k}^{4} a_{k}^{(2)} \\
& +6 \gamma \sigma_{k}\left(\sigma_{k}+1\right)^{3} q_{k} h_{k}^{4} b_{k}^{(1)} \\
& +\left(6\left(\sigma_{k}-1\right)\left(\sigma_{k}+1\right)^{2} q_{k}+6\left(\sigma_{k}+1\right)^{3}\right. \\
& \left.\left.\times\left(r_{k}-p_{k}\right)\right) h_{k}\right) a_{k}-6\left(\sigma_{k}+1\right)^{3} \\
& \times\left(\sigma_{k} p_{k}+r_{k}\right) h_{k}^{2} a_{k}^{(1)}+3 \sigma_{k}\left(\sigma_{k}+2\right)\left(\sigma_{k}+1\right) \\
& \times r_{k} h_{k}^{3} a_{k}^{(2)}+6\left(\sigma_{k}+1\right)^{2} \sigma_{k}\left(\sigma_{k} p_{k}-r_{k}\right) h_{k}^{3} b_{k}^{(1)} \\
& +3 \sigma_{k}\left(\sigma_{k}+1\right)^{2}\left(\sigma_{k}^{2} p_{k}+r_{k}\right) h_{k}^{4} b_{k}^{(2)} \\
& -a_{k}^{(3)} \sigma_{k}\left(\sigma_{k}+2\right)\left(\sigma_{k}+1\right) r_{k} h_{k}^{4} \\
& \left.+6 \sigma_{k} b_{k}\left(\sigma_{k}+1\right)^{2} q_{k} h_{k}^{2}\right) \times\left(72 \sigma_{k}\left(\sigma_{k}+1\right)^{2}\right)^{-1}, \\
& \widetilde{R}_{k}^{21}=-\frac{h_{k}^{4}}{24}\left(2\left(\sigma_{k}+1\right) \gamma q_{k} a_{k} g_{k}^{(1)}+\left(\sigma_{k}^{2} p_{k}+r_{k}\right) g_{k}^{(2)}\right) \\
& -\frac{h_{k}^{3}}{12}\left(\sigma_{k} p_{k}-r_{k}\right) g_{k}^{(1)}-\frac{h_{k}^{2}}{12}\left(q_{k}+r_{k}+p_{k}\right) g_{k} \text {. }
\end{aligned}
$$

The modified scheme (24) is free from the terms $1 /(k \pm 1)$, hence easily solved for $k=1(1) n$. The difference equation (24) along with the boundary conditions (4) gives a $2 n \times 2 n$ linear system of equations for the unknowns $U_{k}, V_{k}, k=1(1) n$. The resulting block tridiagonal system can be easily solved using block Gauss-Seidel algorithm.

\section{Extension to Sixth-Order Differential Equations}

The proposed method can be easily extended to the sixthorder differential equations:

$$
\begin{gathered}
u^{(6)}(r)=f\left(r, u(r), u^{(1)}(r), u^{(2)}(r), u^{(3)}(r), u^{(4)}(r),\right. \\
\left.u^{(5)}(r)\right), \quad \alpha<r<\beta
\end{gathered}
$$

subject to the necessary boundary conditions:

$$
\begin{array}{lll}
u(\alpha)=\alpha_{0}, & u^{(2)}(\alpha)=\alpha_{1}, & u^{(4)}(\alpha)=\alpha_{2}, \\
u(\beta)=\beta_{0}, & u^{(2)}(\beta)=\beta_{1}, & u^{(4)}(\beta)=\beta_{2}
\end{array}
$$

or equivalently,

$$
\begin{gathered}
u^{(2)}(r)=v(r), \\
v^{(2)}(r)=w(r), \\
w^{(2)}(r)=f\left(r, u, u^{(1)}(r), v(r), v^{(1)}(r), w(r), w^{(1)}(r)\right), \\
\alpha<r<\beta,
\end{gathered}
$$

subject to the natural boundary conditions:

$$
\begin{array}{lll}
u(\alpha)=\alpha_{0}, & v(\alpha)=\alpha_{1}, & w(\alpha)=\alpha_{2}, \\
u(\beta)=\beta_{0}, & v(\beta)=\beta_{1}, & w(\beta)=\beta_{2} .
\end{array}
$$

We outline the similar algorithm for (28) as follows:

$$
\begin{aligned}
\widetilde{U}_{k}^{\prime} & =\frac{U_{k+1}-\left(1-\sigma_{k}^{2}\right) U_{k}-\sigma_{k}^{2} U_{k-1}}{h_{k} \sigma_{k}\left(1+\sigma_{k}\right)}, \\
\widetilde{V}_{k}^{\prime} & =\frac{V_{k+1}-\left(1-\sigma_{k}^{2}\right) V_{k}-\sigma_{k}^{2} V_{k-1}}{h_{k} \sigma_{k}\left(1+\sigma_{k}\right)}, \\
\widetilde{W}_{k}^{\prime} & =\frac{W_{k+1}-\left(1-\sigma_{k}^{2}\right) W_{k}-\sigma_{k}^{2} W_{k-1}}{h_{k} \sigma_{k}\left(1+\sigma_{k}\right)}, \\
\widetilde{U}_{k+1}^{\prime} & =\frac{\left(1+2 \sigma_{k}\right) U_{k+1}-\left(1+\sigma_{k}\right)^{2} U_{k}+\sigma_{k}^{2} U_{k-1}}{h_{k} \sigma_{k}\left(1+\sigma_{k}\right)}, \\
\widetilde{V}_{k+1}^{\prime}= & \frac{\left(1+2 \sigma_{k}\right) V_{k+1}-\left(1+\sigma_{k}\right)^{2} V_{k}+\sigma_{k}^{2} V_{k-1}}{h_{k} \sigma_{k}\left(1+\sigma_{k}\right)}, \\
\widetilde{W}_{k+1}^{\prime}= & \frac{\left(1+2 \sigma_{k}\right) W_{k+1}-\left(1+\sigma_{k}\right)^{2} W_{k}+\sigma_{k}^{2} W_{k-1}}{h_{k} \sigma_{k}\left(1+\sigma_{k}\right)},
\end{aligned}
$$




$$
\begin{aligned}
\widetilde{U}_{k-1}^{\prime} & =\frac{-U_{k+1}+\left(1+\sigma_{k}\right)^{2} U_{k}-\sigma_{k}\left(2+\sigma_{k}\right) U_{k-1}}{h_{k} \sigma_{k}\left(1+\sigma_{k}\right)}, \\
\widetilde{V}_{k-1}^{\prime} & =\frac{-V_{k+1}+\left(1+\sigma_{k}\right)^{2} V_{k}-\sigma_{k}\left(2+\sigma_{k}\right) V_{k-1}}{h_{k} \sigma_{k}\left(1+\sigma_{k}\right)}, \\
\widetilde{W}_{k-1}^{\prime} & =\frac{-W_{k+1}+\left(1+\sigma_{k}\right)^{2} W_{k}-\sigma_{k}\left(2+\sigma_{k}\right) W_{k-1}}{h_{k} \sigma_{k}\left(1+\sigma_{k}\right)} \\
\widetilde{f}_{k \pm 1} & =f\left(r_{k \pm 1}, U_{k \pm 1}, \widetilde{U}_{k \pm 1}^{\prime}, V_{k \pm 1}, \widetilde{V}_{k \pm 1}^{\prime}, W_{k \pm 1}, \widetilde{W}_{k \pm 1}^{\prime}\right), \\
\widehat{U}_{k}^{\prime} & =\widetilde{U}_{k}^{\prime}+\gamma h_{k}\left(V_{k+1}-V_{k-1}\right) \\
\widehat{V}_{k}^{\prime} & =\widetilde{V}_{k}^{\prime}+\gamma h_{k}\left(W_{k+1}-W_{k-1}\right), \\
\widehat{W}_{k}^{\prime} & =\widetilde{W}_{k}^{\prime}+\gamma h_{k}\left(\widetilde{f}_{k+1}-\widetilde{f}_{k-1}\right),
\end{aligned}
$$

where the values of $\gamma$ are same as obtained in Section 2.

Then, the $O\left(h_{k}^{3}\right)$-approximations for (26) or (28) can be obtained by the following relations for $k=1(1) n$ :

$$
\begin{aligned}
& U_{k+1}-\left(1+\sigma_{k}\right) U_{k}+\sigma_{k} U_{k-1} \\
& +\frac{h^{2}}{12}\left(p_{k} V_{k+1}+q_{k} V_{k}+r_{k} V_{k-1}\right)=O\left(h_{k}^{5}\right), \\
& V_{k+1}-\left(1+\sigma_{k}\right) V_{k}+\sigma_{k} V_{k-1} \\
& +\frac{h^{2}}{12}\left(p_{k} W_{k+1}+q_{k} W_{k}+r_{k} W_{k-1}\right)=O\left(h_{k}^{5}\right), \\
& W_{k+1}-\left(1+\sigma_{k}\right) W_{k}+\sigma_{k} W_{k-1} \\
& +\frac{h_{k}^{2}}{12}\left(p_{k} \tilde{f}_{k}+q_{k} \widehat{f}_{k}+r_{k} \tilde{f}_{k-1}\right)=O\left(h_{k}^{5}\right) .
\end{aligned}
$$

The boundary conditions (27) are used to obtain values at $k \pm 1$ for $k=1$ and $n$, respectively. The numerical scheme may be implemented by neglecting $O\left(h_{k}^{5}\right)$ terms from (31).

\section{Convergence Analysis}

In this section, we derive the difference scheme of singular problem and investigate the convergence property of the proposed scheme. Consider the model problem

$$
u^{(4)}(r)=a(r) u(r)+g(r), \quad \alpha<r<\beta
$$

or equivalently

$$
\begin{gathered}
u^{(2)}(r)=v(r), \\
v^{(2)}(r)=a(r) u(r)+g(r), \quad \alpha<r<\beta
\end{gathered}
$$

along with the boundary conditions (2).

If $a(r), g(r) \in C^{2}[\alpha, \beta]$ and $a(r)>0$ on $[\alpha, \beta]$, then the boundary value problem (33) has a unique solution. Under these assumptions (see, [27]), there are constants $\underline{a}>0$ and $\underline{g}$ such that $\underline{a}=\min _{k}\left\{a_{k}, a_{k}^{(1)}, a_{k}^{(2)}\right\}, g=\min _{k}\left\{g_{k}, g_{k}^{(1)}, g_{k}^{(2)}\right\}$.
For the convergence, the coefficients $p_{k}, q_{k}$, and $r_{k}$ associated with (5) and (15) must be negative (see, [22]), from which we obtain the condition $\left|\sigma_{k}-\sqrt{5} / 2\right|<1 / 2$.

Now applying the methods (5) and (15) to (33) and using the similar technique discussed in Section 3 for singular coefficients $a(r)$ and $g(r)$, we obtain the following system of difference equations:

$$
\begin{array}{r}
\mathbf{P}_{k} \mathbf{Z}_{k-1}+\mathbf{Q}_{k} \mathbf{Z}_{k}+\mathbf{R}_{k} \mathbf{Z}_{k+1}=\mathbf{S}_{k}+\mathbf{T}_{k}\left(h_{k}\right), \\
k=1(1) n,
\end{array}
$$

where

$$
\mathbf{P}_{k}=\left[\begin{array}{cc}
-\sigma_{k} & \frac{h_{k}^{2}}{12} \sigma_{k}\left(1+\sigma_{k}-\sigma_{k}^{2}\right) \\
\frac{h_{k}^{2}}{12} \sigma_{k}\left(1+\sigma_{k}-\sigma_{k}^{2}\right) & \\
\times\left(a_{k}+h_{k} a_{k}^{(1)}\right) & -\sigma_{k}
\end{array}\right],
$$

$\mathbf{Q}_{k}=\left(\sigma_{k}+1\right)$

$$
\begin{aligned}
& \times\left[\begin{array}{cc}
1 & \frac{h_{k}^{2}}{12}\left(\sigma_{k}^{2}+3 \sigma_{k}+1\right) \\
\frac{h_{k}^{2}}{24}\left(2\left(\sigma_{k}^{2}+3 \sigma_{k}+1\right) a_{k}\right. & \\
+4 h_{k} \sigma_{k}\left(\sigma_{k}-1\right) a_{k}^{(1)} & \\
+h_{k}^{2} \sigma_{k}\left(\sigma_{k}^{2}-\sigma_{k}\right. & \\
\left.+1) a_{k}^{(2)}\right) & 1
\end{array}\right], \\
& \mathbf{R}_{k}=\left[\begin{array}{cc}
-1 & \frac{h_{k}^{2}}{12}\left(\sigma_{k}^{2}+\sigma_{k}-1\right) \\
\frac{h_{k}^{2}}{12}\left(\left(\sigma_{k}^{2}+\sigma_{k}-1\right) a_{k}\right. & -1 \\
\left.+h_{k} \sigma_{k}\left(\sigma_{k}^{2}-\sigma_{k}+1\right) a_{k}^{(1)}\right) &
\end{array}\right] \text {, } \\
& \mathbf{S}_{k}=\left[0, \frac{h_{k}^{2}}{12}\left(\left(\sigma_{k}^{3}-\sigma_{k}^{2}-\sigma_{k}\right) g_{k}^{(2)}-\left(\sigma_{k}^{2}+\sigma_{k}-1\right) g_{k}^{(1)}\right.\right. \\
& \left.\left.-\left(\sigma_{k}^{3}+4 \sigma_{k}^{2}+4 \sigma_{k}+1\right) g_{k}\right)\right]^{T} \\
& \mathbf{Z}_{k}=\left[U_{k}, V_{k}\right]^{T}, \quad \mathbf{T}_{k}\left(h_{k}\right)=O\left(h_{k}^{5}\right) .
\end{aligned}
$$

Incorporating the boundary values $U_{0}=\alpha_{0}, V_{0}=\alpha_{1}, U_{n+1}=$ $\beta_{0}$, and $V_{n+1}=\beta_{1}$, the system of difference equations (34) in the matrix-vector form can be written as

$$
\mathbf{M Z}=\mathbf{J}+\mathbf{T}_{k}\left(h_{k}\right)
$$

where $\mathbf{M}=\left[\begin{array}{lll}\mathbf{P}_{k} & \mathbf{Q}_{k} & \mathbf{R}_{k}\end{array}\right]$ is the block tridiagonal matrix, $\mathbf{J}=$ $\left[\mathbf{S}_{1}-\mathbf{P}_{1} \boldsymbol{\alpha}, \mathbf{S}_{2}, \ldots, \mathbf{S}_{n-1}, \mathbf{S}_{n}-\mathbf{R}_{n} \boldsymbol{\beta}\right]^{T}, \boldsymbol{\alpha}=\left[\alpha_{0}, \alpha_{1}\right]^{T}$, and $\boldsymbol{\beta}=$ $\left[\beta_{0}, \beta_{1}\right]^{T}$.

Let $z_{k}=\left[u_{k}, v_{k}\right]^{T}, k=1(1) n$, and $\mathbf{z}=\left[z_{1}, \ldots, z_{n}\right] \cong \mathbf{Z}$, which satisfies

$$
\mathbf{M z}=\mathbf{J} .
$$


Let $\boldsymbol{\varepsilon}=\left[\varepsilon_{1}, \varepsilon_{2}, \ldots, \varepsilon_{n}\right]^{T}$ be the discretization error vector, and let $\varepsilon_{k}=Z_{k}-z_{k}, k=1(1) n$ be the discretization errors at the node $r_{k}$. Subtracting (37) from (36), we obtain the error equation:

$$
\mathbf{M} \boldsymbol{\varepsilon}=\mathbf{T}_{k}\left(h_{k}\right) .
$$

Also, we obtain

$$
\begin{aligned}
& \left\|\mathbf{P}_{k}\right\|_{\infty}=\max _{2 \leq k \leq n}\left\{\begin{array}{c}
\sigma_{k}+\frac{h_{k}^{2}}{12} \sigma_{k}\left|1+\sigma_{k}-\sigma_{k}^{2}\right|, \\
\sigma_{k}+\frac{h_{k}^{2}}{12} \sigma_{k}\left|1+\sigma_{k}-\sigma_{k}^{2}\right| \cdot\left|a_{k}+h_{k} a_{k}^{(1)}\right|,
\end{array}\right. \\
& \left\|\mathbf{R}_{k}\right\|_{\infty}=\max _{1 \leq k \leq n-1}\left\{\begin{array}{c}
1+\frac{h_{k}^{2}}{12}\left|\sigma_{k}^{2}+\sigma_{k}-1\right|, \\
1+\frac{h_{k}^{2}}{12} \mid\left(\sigma_{k}^{2}+\sigma_{k}-1\right) a_{k} \\
+h_{k} \sigma_{k}\left(\sigma_{k}^{2}-\sigma_{k}+1\right) a_{k}^{(1)} \mid .
\end{array}\right.
\end{aligned}
$$

Thus for sufficiently small $h_{k}$ or equivalently as $h_{k} \rightarrow 0$, we obtain the relations $\left\|\mathbf{P}_{k}\right\|_{\infty}=\sigma_{k}, k=2(1) n$ and $\left\|\mathbf{R}_{k}\right\|_{\infty}=1$, $k=1(1) n-1$. Hence, the graph $\mathbf{G}(\mathbf{M})$ of the matrix $\mathbf{M}$ is strongly connected, and thus the matrix $\mathbf{M}$ is irreducible (see, [28]).

Further, let $\Sigma_{l}$ be the sum of the $l$ th row sum of the matrix $\mathbf{M}$; then we have the following.

$$
\text { For } l=1 \text {, }
$$

$$
\begin{aligned}
\Sigma_{l}= & \sigma_{l}+\frac{h_{l}^{2}}{12} \sigma_{l}\left(\sigma_{l}^{2}+5 \sigma_{l}+5\right), \\
\Sigma_{l+1}= & \sigma_{l}+\frac{h_{l}^{2}}{12} a_{l} \sigma_{l}\left(\sigma_{l}^{2}+5 \sigma_{l}+5\right) \\
& +\frac{h_{l}^{3}}{12} a_{l}^{(1)} \sigma_{l}\left(3 \sigma_{l}^{2}-\sigma_{l}-1\right)+\frac{h_{l}^{4}}{24} a_{l}^{(2)} \sigma_{l}\left(\sigma_{l}^{3}+1\right) .
\end{aligned}
$$

For $l=3(2) 2 n-3$,

$$
\begin{gathered}
\Sigma_{l}=\frac{h_{l}^{2}}{2} \sigma_{l}\left(\sigma_{l}+1\right), \\
\Sigma_{l+1}=\frac{h_{l}^{2}}{2} a_{l} \sigma_{l}\left(\sigma_{l}+1\right)+\frac{h_{l}^{3}}{6} a_{l}^{(1)} \sigma_{l}\left(\sigma_{l}^{2}-1\right) \\
+\frac{h_{l}^{4}}{24} a_{l}^{(2)} \sigma_{l}\left(\sigma_{l}^{3}+1\right) .
\end{gathered}
$$

For $l=2 n-1$,

$$
\begin{gathered}
\Sigma_{l}=1+\frac{h_{l}^{2}}{12}\left(5 \sigma_{l}^{2}+5 \sigma_{l}+1\right), \\
\Sigma_{l+1}=1+\frac{h_{l}^{2}}{12} a_{l}\left(5 \sigma_{l}^{2}+5 \sigma_{l}+1\right)+\frac{h_{l}^{3}}{12} a_{l}^{(1)} \sigma_{l}\left(\sigma_{l}^{2}+\sigma_{l}-3\right) \\
+\frac{h_{l}^{4}}{24} a_{l}^{(2)} \sigma_{l}\left(\sigma_{l}^{3}+1\right) .
\end{gathered}
$$

This implies the following.
For $l=1$,

$$
\begin{gathered}
\Sigma_{l}=\sigma_{l}+\frac{h_{l}^{2}}{12} \sigma_{l}\left(\sigma_{l}^{2}+5 \sigma_{l}+5\right), \\
\Sigma_{l+1} \geq \sigma_{l}+\frac{h_{l}^{2}}{12} \underline{a} \sigma_{l}\left(\sigma_{l}^{2}+5 \sigma_{l}+5\right) \\
+\frac{h_{l}^{3}}{12} \underline{a} \sigma_{l}\left(3 \sigma_{l}^{2}-\sigma_{l}-1\right)+\frac{h_{l}^{4}}{24} \underline{a} \sigma_{l}\left(\sigma_{l}^{3}+1\right) .
\end{gathered}
$$

For $l=3(2) 2 n-3$,

$$
\begin{gathered}
\Sigma_{l}=\frac{h_{l}^{2}}{2} \sigma_{l}\left(\sigma_{l}+1\right), \\
\Sigma_{l+1} \geq \frac{h_{l}^{2}}{2} \underline{a} \sigma_{l}\left(\sigma_{l}+1\right)+\frac{h_{l}^{3}}{6} \underline{a} \sigma_{l}\left(\sigma_{l}^{2}-1\right) \\
+\frac{h_{l}^{4}}{24} \underline{a} \sigma_{l}\left(\sigma_{l}^{3}+1\right) .
\end{gathered}
$$

For $l=2 n-1$,

$$
\begin{gathered}
\Sigma_{l}=1+\frac{h_{l}^{2}}{12}\left(5 \sigma_{l}^{2}+5 \sigma_{l}+1\right), \\
\Sigma_{l+1} \geq 1+\frac{h_{l}^{2}}{12} \underline{a}\left(5 \sigma_{l}^{2}+5 \sigma_{l}+1\right) \\
+\frac{h_{l}^{3}}{12} \underline{a} \sigma_{l}\left(\sigma_{l}^{2}+\sigma_{l}-3\right)+\frac{h_{l}^{4}}{24} \underline{a} \sigma_{l}\left(\sigma_{l}^{3}+1\right),
\end{gathered}
$$

For sufficiently small value of $h_{k}$, that is, in the limiting case as $h_{k} \rightarrow 0$, we obtain

$$
\begin{aligned}
& \Sigma_{l} \geq \sigma_{l}>0, \quad l=1,2, \\
& \Sigma_{l} \geq 0, \quad l=3(1) 2 n-2, \\
& \Sigma_{l} \geq 1>0, \quad l=2 n-1,2 n .
\end{aligned}
$$

Hence we find that $\mathbf{M}$ is monotone (see, $[29,30])$. Consequently $\mathbf{M}^{-1}$ exists, and $\mathbf{M}^{-1} \geq 0$.

Let $\mathbf{M}_{i, l}^{-1}$ be the $(i, l)$ th element of $\mathbf{M}^{-1}$, and we define

$$
\begin{aligned}
& \left\|\mathbf{M}^{-1}\right\|=\max _{1 \leq i \leq 2 n} \sum_{l=1}^{2 n}\left|\mathbf{M}_{i, l}^{-1}\right|, \\
& \|\mathbf{T}\|=\max _{1 \leq l \leq 2 n} \sum_{l=1}^{2 n}\left|\mathbf{T}_{l}\left(h_{l}\right)\right|=O\left(h_{l}^{3}\right) .
\end{aligned}
$$

From the theory of matrix, we know that

$$
\sum_{l=1}^{2 n} \mathbf{M}_{i, l}^{-1} \Sigma_{l}=1, \quad 1 \leq i \leq 2 n .
$$

Thus the following bounds can be estimated with the help of series expansions. 
For $l=1$,

$$
\begin{aligned}
\mathbf{M}_{i, l}^{-1} \leq \Sigma_{l}^{-1}=\frac{1}{\sigma_{l}} & -\frac{h_{l}^{2}}{12 \sigma_{l}}\left(\sigma_{l}^{2}+5 \sigma_{l}+5\right)+O\left(h_{l}^{4}\right), \\
\mathbf{M}_{i, l+1}^{-1} \leq \Sigma_{l+1}^{-1} \leq & \frac{1}{\sigma_{l}}-\frac{h_{l}^{2} \underline{a}}{12 \sigma_{l}}\left(\sigma_{l}^{2}+5 \sigma_{l}+5\right) \\
& -\frac{h_{l}^{3} \underline{a}}{12 \sigma_{l}}\left(3 \sigma_{l}^{2}-\sigma_{l}-1\right)+O\left(h_{l}^{4}\right) .
\end{aligned}
$$

For $l=3(2) 2 n-3$,

$$
\begin{aligned}
\mathbf{M}_{i, l}^{-1} \leq \min _{l} \Sigma_{l}^{-1}=\frac{2}{h_{l}^{2} \sigma_{l}\left(\sigma_{l}+1\right)} & \\
\mathbf{M}_{i, l+1}^{-1} \leq \min _{l} \Sigma_{l+1}^{-1} \leq & \frac{2}{h_{l}^{2} \underline{a} \sigma_{l}\left(\sigma_{l}+1\right)} \\
& -\frac{2\left(\sigma_{l}-1\right)}{3 h_{l} \underline{a} \sigma_{l}\left(\sigma_{l}+1\right)}+\frac{\sigma_{l}^{2}-5 \sigma_{l}+1}{18 \underline{a} \sigma_{l}\left(\sigma_{l}+1\right)} \\
& +O\left(h_{l}\right) .
\end{aligned}
$$

For $l=2 n-1$,

$$
\begin{aligned}
\mathbf{M}_{i, l}^{-1} \leq \Sigma_{l}^{-1}=1 & -\frac{h_{l}^{2}}{12}\left(5 \sigma_{l}^{2}+5 \sigma_{l}+1\right)+O\left(h_{l}^{4}\right), \\
\mathbf{M}_{i, l+1}^{-1} \leq \Sigma_{l+1}^{-1} \leq 1 & -\frac{h_{l}^{2} \underline{a}}{12}\left(5 \sigma_{l}^{2}+5 \sigma_{l}+1\right) \\
& -\frac{h_{l}^{3} \underline{a}}{12} \sigma_{l}\left(\sigma_{l}^{2}+\sigma_{l}-3\right)+O\left(h_{l}^{4}\right) .
\end{aligned}
$$

With the help of (48), we obtain the following bounds:

$$
\begin{aligned}
\left\|\mathbf{M}^{-1}\right\| \leq & \frac{2(\underline{a}+1)}{h_{l}^{2} \underline{a} \sigma_{l}\left(\sigma_{l}+1\right)}-\frac{2\left(\sigma_{l}-1\right)}{3 h_{l} \underline{a} \sigma_{l}\left(\sigma_{l}+1\right)} \\
& +\frac{\left(\sigma_{l}^{2}-5 \sigma_{l}+1+36 \underline{a}\left(\sigma_{l}+1\right)^{2}\right)}{18 \underline{a} \sigma_{l}\left(\sigma_{l}+1\right)}+O\left(h_{l}\right) .
\end{aligned}
$$

From (38) and (52), we obtain the following error estimates:

$$
\|\varepsilon\| \leq\left\|\mathbf{M}^{-1}\right\| \cdot\left\|\mathbf{T}_{l}\left(h_{l}\right)\right\| \leq O\left(h_{l}^{3}\right) .
$$

This proves the third-order convergence of the proposed method. We generalize the above results in the following theorem.

Theorem 1. The method given by (15) for the numerical solution of fourth-order singular differential equation (1) with sufficiently small $h_{k}$ and $\left|\sigma_{k}-\sqrt{5} / 2\right|<1 / 2, \sigma_{k} \neq 1$ gives a thirdorder convergent solution.

\section{Computational Illustrations}

To illustrate the geometric mesh finite difference method, we have solved both linear and nonlinear problems. The boundary conditions may be obtained from the analytical solution as a test procedure. The numerical accuracy of results are tested using maximum absolute errors and root mean square errors with the error tolerance being $\leq 10^{-15}$. For the simplicity in computation, we choose $\sigma_{k}=\sigma=$ constant, for $k=1(1) n$ and define the geometric mesh as follows ([24]):

$$
\begin{gathered}
x_{0}=\alpha, \\
h_{1}= \begin{cases}\frac{(\beta-\alpha)(1-\sigma)}{\left(1-\sigma^{n+1}\right)}, & \sigma<1, \\
\frac{(\beta-\alpha)(\sigma-1)}{\left(\sigma^{n+1}-1\right)}, & \sigma>1 .\end{cases}
\end{gathered}
$$

The subsequent mesh spacing is determined by $h_{k+1}=\sigma h_{k}$, $k=1(1) n$. If the boundary value problems exhibit layer behaviour near the left boundary (see, [21]), the solution value can be captured by choosing $\sigma>1$. If the layer occurs at the right boundary, we choose $\sigma<1$. If the layer occurs in the interior region, then mesh in the first half of the interval may be arranged by choosing $\sigma>1$ and second half of the interval by choosing $\sigma<1$.

All the numerical computations are performed using long double length arithmetic in $C$ under Linux operating system with $2 \mathrm{~GB}$ operational memory.

Example 1. Consider the fourth-order linear problem (see, [31]) in the polar form:

$$
\begin{aligned}
\Delta^{4} u(r) & \equiv\left(\frac{d^{2}}{d r^{2}}+\frac{\lambda}{r} \frac{d}{d r}\right)^{2} u(r) \\
& =\left(1+\frac{2 \lambda}{r}+\frac{\lambda(\lambda-2)}{r^{2}}-\frac{\lambda(\lambda-2)}{r^{3}}\right) e^{r}, \\
& 0<r<1 .
\end{aligned}
$$

The analytical solution is $u(r)=e^{r}$. The errors estimates for various values of $n$ are reported in Tables 1 and 2 for uniform mesh $(\sigma=1)$ and geometric mesh $(\sigma \neq 1)$, respectively.

Example 2. Consider the boundary value problems that arise from time-dependent Navier-Stokes equation (see, [32]) for axis symmetric flow of an incompressible fluid contained between infinite disks

$$
\begin{aligned}
u^{(4)}(r)= & \lambda u(r) u^{(2)}(r)-\lambda\left(r^{2}-1\right) \\
& \times\left(1+4 r+r^{2}\right) e^{2 r}-\left(11+8 r+r^{2}\right) e^{r}, \\
& 0<r<1 .
\end{aligned}
$$

The analytical solution is $u(r)=\left(1-r^{2}\right) e^{r}$. The errors estimates are reported in Table 3 for various values of $n$ and $\lambda=10^{3}$. 
TABLE 1: The maximum absolute errors for Example 1.

\begin{tabular}{lcccc}
\hline & \multicolumn{2}{c}{$\lambda=1$} & \multicolumn{2}{c}{$\lambda=2$} \\
& 1 & 0.9937 & 1 & 0.996759 \\
\hline 8 & $0.866 e-04$ & $0.772 e-04$ & $0.290 e-05$ & $0.271 e-05$ \\
16 & $0.223 e-04$ & $0.176 e-04$ & $0.190 e-05$ & $0.170 e-05$ \\
32 & $0.123 e-04$ & $0.730 e-05$ & $0.640 e-08$ & $0.246 e-08$ \\
64 & $0.615 e-06$ & $0.348 e-08$ & $0.365 e-09$ & $0.882 e-10$ \\
\hline
\end{tabular}

TABLE 2: The root mean square errors for Example 1.

\begin{tabular}{lcccc}
\hline & \multicolumn{2}{c}{$\lambda=1$} & \multicolumn{2}{c}{$\lambda=2$} \\
$n$ & \multicolumn{2}{c}{$\sigma$} & 1 & 0.996759 \\
\hline 8 & $0.664 e-04$ & $0.590 e-04$ & $0.219 e-05$ & $0.205 e-05$ \\
16 & $0.166 e-04$ & $0.131 e-04$ & $0.139 e-05$ & $0.125 e-05$ \\
32 & $0.877 e-05$ & $0.517 e-05$ & $0.419 e-08$ & $0.239 e-08$ \\
64 & $0.417 e-06$ & $0.214 e-08$ & $0.214 e-09$ & $0.483 e-10$ \\
\hline
\end{tabular}

TABLE 3: The maximum absolute errors and root mean square errors for Example 2.

\begin{tabular}{lcccc}
\hline$n$ & \multicolumn{4}{c}{$\sigma$} \\
& 1 & 0.998 & 1 & 0.998 \\
\hline 8 & $0.302 e-05$ & $0.255 e-05$ & $0.235 e-05$ & $0.199 e-05$ \\
16 & $0.238 e-06$ & $0.169 e-06$ & $0.179 e-06$ & $0.128 e-06$ \\
32 & $0.167 e-07$ & $0.751 e-08$ & $0.124 e-07$ & $0.557 e-08$ \\
64 & $0.111 e-08$ & $0.919 e-10$ & $0.819 e-09$ & $0.649 e-10$ \\
\hline
\end{tabular}

TABLE 4: The maximum absolute errors for Example 3.

\begin{tabular}{lcccc}
\hline \multicolumn{2}{c}{$\lambda=1$} & \multicolumn{2}{c}{$\lambda=2$} \\
& \multicolumn{1}{c}{1.11} & 1 & 0.994 \\
\hline 8 & $0.338 e-03$ & $0.189 e-03$ & $0.322 e-06$ & $0.268 e-06$ \\
16 & $0.102 e-03$ & $0.292 e-04$ & $0.261 e-07$ & $0.183 e-07$ \\
32 & $0.337 e-04$ & $0.481 e-05$ & $0.186 e-08$ & $0.847 e-09$ \\
64 & $0.111 e-04$ & $0.662 e-06$ & $0.125 e-09$ & $0.126 e-10$ \\
\hline
\end{tabular}

TABLE 5: The root mean square errors for Example 3.

\begin{tabular}{lcccc}
\hline & \multicolumn{2}{c}{$\lambda=1$} & \multicolumn{2}{c}{$\lambda=2$} \\
& \multicolumn{2}{c}{$\sigma$} & 1 & 0.994 \\
\hline 8 & $0.255 e-03$ & $0.140 e-03$ & $0.245 e-06$ & $0.202 e-06$ \\
16 & $0.742 e-04$ & $0.197 e-04$ & $0.193 e-07$ & $0.134 e-07$ \\
32 & $0.240 e-04$ & $0.270 e-05$ & $0.136 e-08$ & $0.589 e-09$ \\
64 & $0.782 e-05$ & $0.243 e-06$ & $0.900 e-10$ & $0.834 e-11$ \\
\hline
\end{tabular}

TABLE 6: The maximum absolute errors and root mean square errors for Example 4.

\begin{tabular}{lcccc}
\hline$n$ & \multicolumn{4}{c}{$\sigma$} \\
& 1 & 0.9991 & 1 & 0.9991 \\
\hline 8 & $0.380 e-07$ & $0.302 e-07$ & $0.291 e-07$ & $0.230 e-07$ \\
16 & $0.301 e-08$ & $0.186 e-08$ & $0.222 e-08$ & $0.134 e-08$ \\
32 & $0.210 e-09$ & $0.587 e-10$ & $0.153 e-09$ & $0.373 e-10$ \\
64 & $0.127 e-10$ & $0.913 e-11$ & $0.912 e-11$ & $0.654 e-11$ \\
\hline
\end{tabular}

Example 3. Consider the sixth-order linear singular problem:

$$
\begin{aligned}
\Delta^{6} u(r) \equiv & \left(\frac{d^{2}}{d r^{2}}+\frac{\lambda}{r} \frac{d}{d r}\right)^{3} u(r) \\
= & \left(1+\frac{3 \lambda(\lambda-2)}{r^{2}}+\frac{3 \lambda(\lambda-2)(\lambda-4)}{r^{4}}\right) \sinh (r) \\
& +\frac{\lambda}{r}\left(3+\frac{(\lambda-2)(\lambda-7)}{r^{2}}\right. \\
& \left.+\frac{3(\lambda-2)(\lambda-4)}{r^{4}}\right) \cosh (r), \quad 0<r<1 .
\end{aligned}
$$

The analytical solution is $u(r)=\sinh (r)$. The errors estimates for various values of $n$ are reported in Tables 4 and 5 for uniform mesh $(\sigma=1)$ and geometric mesh $(\sigma \neq 1)$, respectively.

Example 4. Consider the nonlinear problem

$$
\begin{aligned}
u^{(6)}(r)=\lambda( & u(r) u^{(2)}(r)+u^{(2)}(r) u^{(4)}(r) \\
& \left.+u(r) u^{(4)}(r)\right)+\sinh (r)(1-3 \lambda \sinh (r)), \\
& 0<r<1 .
\end{aligned}
$$

The analytical solution is $u(r)=\sinh (r)$. The errors estimates are reported in Table 6 for various values of $n$ and $\lambda=10^{3}$.

\section{Conclusion}

The numerical results confirm that the proposed geometric mesh finite difference scheme converges and applicable to both singular and nonsingular differential equations. The numerical accuracy obtained using geometric mesh shows superiority over corresponding uniform mesh. The optimum mesh ratio parameter within the specified convergent region may be obtained by simulations. We have employed block Gauss-Seidel method to solve the block matrix systems. The method can be extended to general even-order nonlinear differential equations. Application to the proposed scheme to nonlinear singular elliptic problems is an open problem. 


\section{Acknowledgment}

The authors are thankful to the Professor Rüdiger Weiner and unknown referee for their valuable suggestions which improve the quality of paper.

\section{References}

[1] R. M. Hornreich, M. Luban, and S. Shtrikman, "Critical behavior at the onset of $\mathrm{k} \rightarrow$-space instability on the $\lambda$ line," Physical Review Letters, vol. 35, no. 25, pp. 1678-1681, 1975.

[2] M. C. Cross and P. C. Hohenberg, "Pattern formation outside of equilibrium," Reviews of Modern Physics, vol. 65, no. 3, pp. 851-1112, 1993.

[3] A. Leizarowitz and V. J. Mizel, "One dimensional infinitehorizon variational problems arising in continuum mechanics," Archive for Rational Mechanics and Analysis, vol. 106, no. 2, pp. 161-194, 1989.

[4] A. C. Lazer and P. J. Mckenna, "Large-amplitude periodic oscillations in suspension bridges. Some new connections with nonlinear analysis," SIAM Review, vol. 32, no. 4, pp. 537-578, 1990.

[5] Y. Chen and P. J. McKenna, "Traveling waves in a nonlinearly suspended beam: theoretical results and numerical observations," Journal of Differential Equations, vol. 136, no. 2, pp. 325355, 1997.

[6] C. J. Budd, G. W. Hunt, and M. A. Peletier, "Self-similar fold evolution under prescribed end shortening," Mathematical Geology, vol. 31, no. 8, pp. 989-1004, 1999.

[7] C. J. Amick and J. F. Toland, "Homoclinic orbits in the dynamic phase-space analogy of an elastic strut," European Journal of Applied Mathematics, vol. 3, no. 2, pp. 97-114, 1992.

[8] B. Buffoni, A. R. Champneys, and J. F. Toland, "Bifurcation and coalescence of a plethora of homoclinic orbits for a Hamiltonian system," Journal of Dynamics and Differential Equations, vol. 8, no. 2, pp. 221-279, 1996.

[9] N. N. Akhmediev, A. V. Buryak, and M. Karlsson, "Radiationless optical solitons with oscillating tails," Optics Communications, vol. 110, no. 5-6, pp. 540-544, 1994.

[10] A. Doelman and V. Rottschäfer, "Singularly perturbed and nonlocal modulation equations for systems with interacting instability mechanisms," Journal of Nonlinear Science, vol. 7, no. 4, pp. 371-409, 1997.

[11] A. R. Aftabizadeh, "Existence and uniqueness theorems for fourth-order boundary value problems," Journal of Mathematical Analysis and Applications, vol. 116, no. 2, pp. 415-426, 1986.

[12] R. P. Agarwal and P. R. Krishnamoorthy, "Boundary value problems for $n$th order ordinary differential equations," Bulletin of the Institute of Mathematics, vol. 7, no. 2, pp. 211-230, 1979.

[13] D. O'Regan, "Solvability of some fourth (and higher) order singular boundary value problems," Journal of Mathematical Analysis and Applications, vol. 161, no. 1, pp. 78-116, 1991.

[14] J. Schröder, "Numerical error bounds for fourth order boundary value problems, simultaneous estimation of $u(x)$ and $u$ " $(x)$," Numerische Mathematik, vol. 44, no. 2, pp. 233-245, 1984.

[15] V. Shanthi and N. Ramanujam, "A numerical method for boundary value problems for singularly perturbed fourthorder ordinary differential equations," Applied Mathematics and Computation, vol. 129, no. 2-3, pp. 269-294, 2002.
[16] R. P. Agarwal and Y. M. Chow, "Iterative methods for a fourth order boundary value problem," Journal of Computational and Applied Mathematics, vol. 10, no. 2, pp. 203-217, 1984.

[17] W. K. Zahra, "A smooth approximation based on exponential spline solutions for nonlinear fourth order two point boundary value problems," Applied Mathematics and Computation, vol. 217, no. 21, pp. 8447-8457, 2011.

[18] J. Rashidinia and M. Ghasemi, "B-spline collocation for solution of two-point boundary value problems," Journal of Computational and Applied Mathematics, vol. 235, no. 8, pp. 2325-2342, 2011.

[19] R. A. Usmani and P. J. Taylor, "Finite difference methods for solving $\left(p(x) y^{\prime \prime}\right)^{\prime \prime}+q(x) y=r(x)$," International Journal of Computer Mathematics, vol. 14, no. 3-4, pp. 277-293, 1983.

[20] D. Britz, Digital Simulation in Electrochemistry, vol. 66 of Lecture Notes in Physics, Springer, Berlin, Germany, 2005.

[21] M. K. Kadalbajoo and D. Kumar, "Geometric mesh FDM for self-adjoint singular perturbation boundary value problems," Applied Mathematics and Computation, vol. 190, no. 2, pp. 16461656, 2007.

[22] M. K. Jain, S. R. K. Iyengar, and G. S. Subramanyam, "Variable mesh methods for the numerical solution of two-point singular perturbation problems," Computer Methods in Applied Mechanics and Engineering, vol. 42, no. 3, pp. 273-286, 1984.

[23] R. K. Mohanty, "A class of non-uniform mesh three point arithmetic average discretization for $y^{\prime \prime}=f\left(x, y, y^{\prime}\right)$ and the estimates of $\mathrm{y}^{\prime}$," Applied Mathematics and Computation, vol.183, no. 1, pp. 477-485, 2006.

[24] N. Jha, "A fifth order accurate geometric mesh finite difference method for general nonlinear two point boundary value problems," Applied Mathematics and Computation, vol. 219, no. 16, pp. 8425-8434, 2013.

[25] S. R. K. Iyengar and P. Jain, "Spline finite difference methods for singular two point boundary value problems," Numerische Mathematik, vol. 50, no. 3, pp. 363-376, 1986.

[26] R. D. Russell and L. F. Shampine, "Numerical methods for singular boundary value problems," SIAM Journal on Numerical Analysis, vol. 12, pp. 13-36, 1975.

[27] W. Gautschi, Numerical Analysis, Birkhause, 2011.

[28] R. S. Varga, Matrix Iterative Analysis, vol. 27 of Springer Series in Computational Mathematics, Springer, Berlin, 2000.

[29] P. Henrici, Discrete Variable Methods in Ordinary Differential Equations, John Wiley \& Sons, New York, NY, USA, 1962.

[30] D. M. Young, Iterative Solution of Large Linear Systems, Academic Press, New York, NY, USA, 1971.

[31] J. Talwar and R. K. Mohanty, "A class of numerical methods for the solution of fourth-order ordinary differential equations in polar coordinates," Advances in Numerical Analysis, vol. 2012, Article ID 626419, 20 pages, 2012.

[32] A. R. Elcrat, "On the radial flow of a viscous fluid between porous disks," Archive for Rational Mechanics and Analysis, vol. 61, no. 1, pp. 91-96, 1976. 


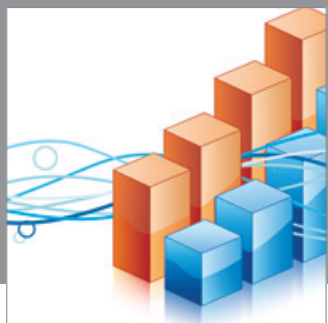

Advances in

Operations Research

mansans

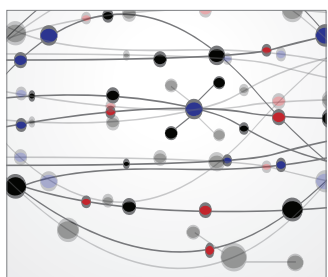

The Scientific World Journal
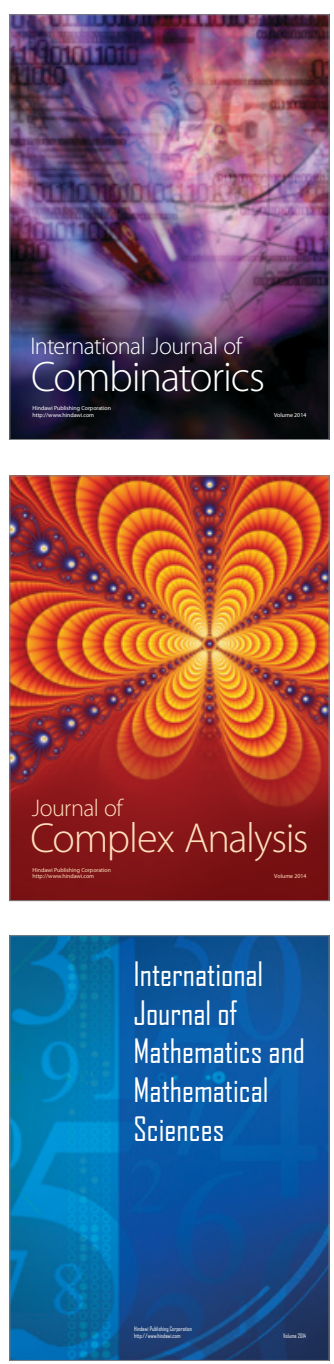
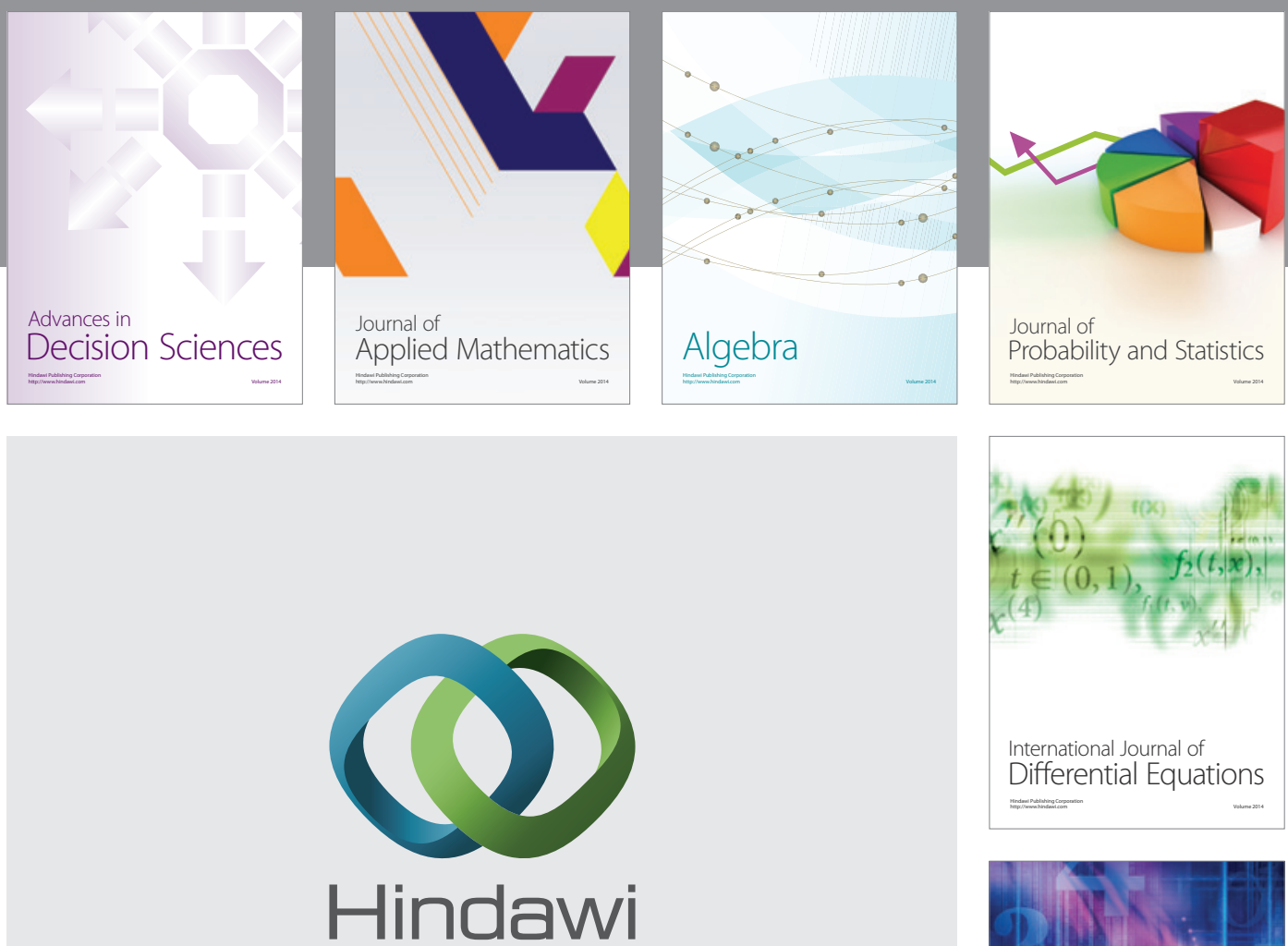

Submit your manuscripts at http://www.hindawi.com
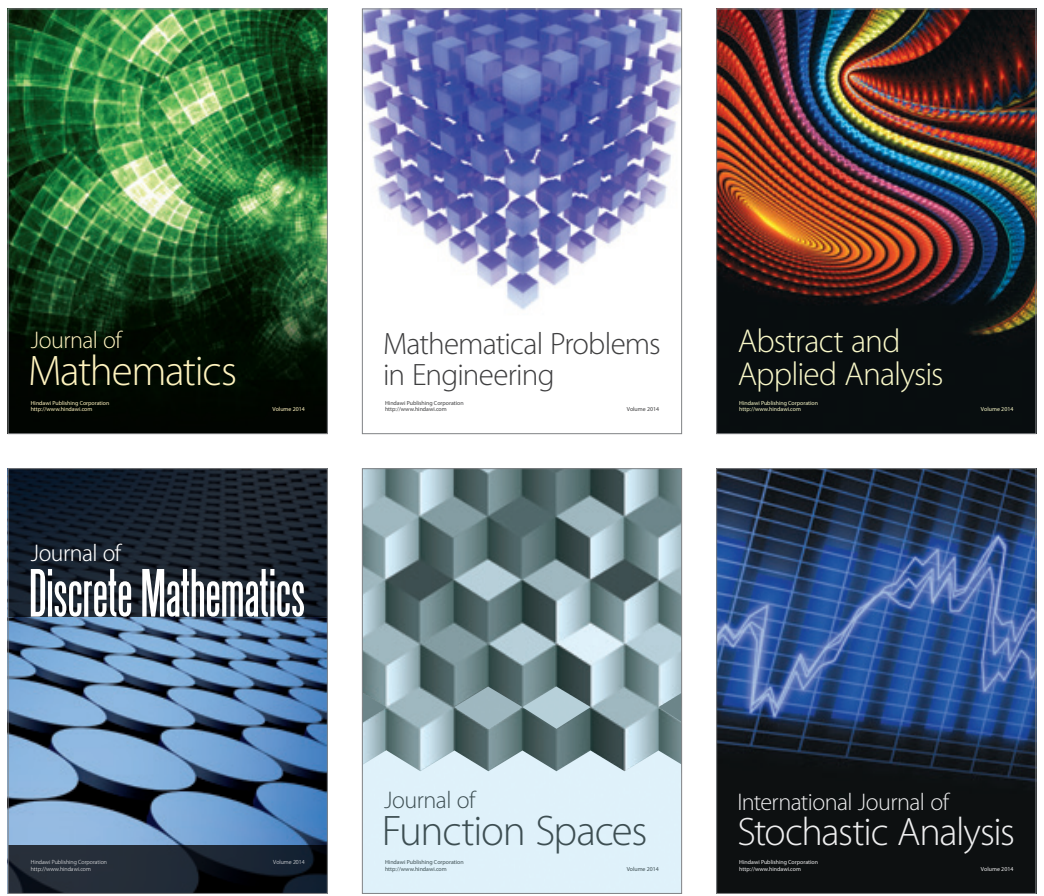

Journal of

Function Spaces

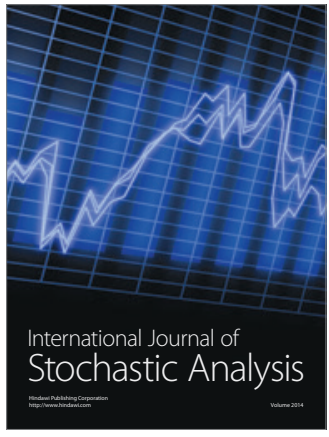

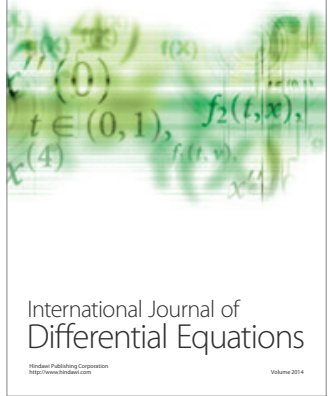
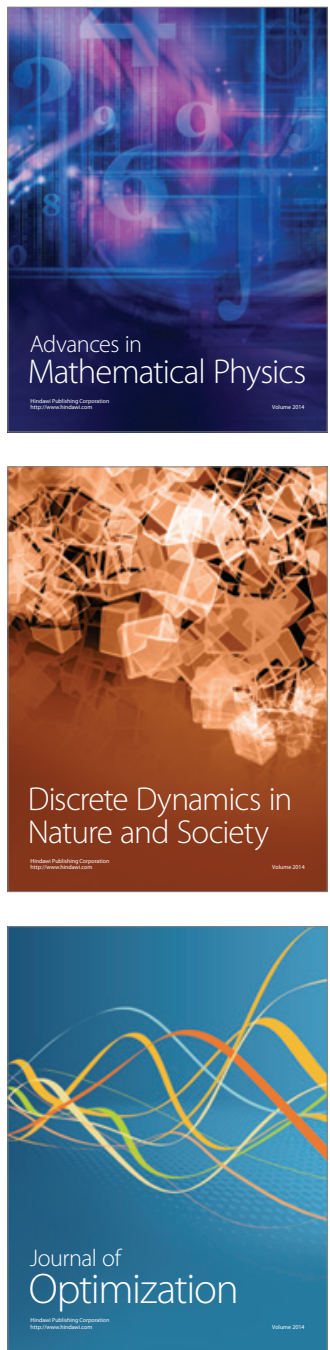